\title{
DISCRETE DIFFERENTIAL FORMS FOR COSMOLOGICAL SPACE-TIMES
}

\author{
RONNY RICHTER AND JÖRG FRAUENDIENER
}

\begin{abstract}
In this article we describe applications of the numerical method of discrete differential forms in computational GR. In particular we consider the initial value problem for vacuum space-times that admit plane gravitational waves. As described in an earlier paper the discrete differential form approach can provide accurate results in spherically symmetric space-times [28]. Moreover it is manifestly coordinate independent.

Here we use the polarised Gowdy solution as a testbed for two numerical schemes. One scheme reproduces that solution very well, in particular it is stable for a comparatively long time and converges quadratically.
\end{abstract}

\section{Introduction}

In an earlier paper on the application of discrete differential forms in numerical General Relativity we described this method for spherically symmetric problems [28]. Here we apply the numerical schemes in the context of $\mathbb{T}^{2}$ symmetric cosmological space-times.

In [28] we used discrete differential forms to calculate the geometry of the domain of dependence of a given initial hypersurface. In that context the domain of dependence was only a small section of the full space-time, which makes it impossible to investigate the longterm behaviour of the numerical schemes unless one is prepared to introduce outgoing boundary conditions. However, this would not allow us to judge the pure evolution algorithm. Thus, we consider space-times which have compact Cauchy surfaces, i.e. cosmological solutions of Einstein's equations.

Thus, we will consider space-times $\mathcal{M}$ with the topology $\Sigma \times \mathbb{R}$, where $\Sigma$ is a closed 3-manifold homeomorphic to the Cauchy surfaces of $\mathcal{M}$. The topology of the Cauchy surface can be controlled quite easily within the discrete differential form approach, because it is given in the very beginning in terms of incidence matrices [6]. Hence, we do not need to worry about outgoing boundary conditions anymore. For instance, if the topology of $\Sigma$ is the 3 -torus $\mathbb{T}^{3}$ then one may equally well use the interpretation that periodic boundary conditions are imposed in each direction. We will not consider the full 3-dimensional case here but as in [28] we will confine ourselves to cases where the problem is effectively $1+1$-dimensional by imposing appropriate symmetries.

An example for a $\mathbb{T}^{2}$ symmetric space-time which admits Cauchy surfaces with topology $\mathbb{T}^{3}$ is the polarised Gowdy solution [15]. This solution can be interpreted as describing gravitational waves in a cosmological universe. In contrast to the static spherically symmetric space-times of [28] this solution is time-dependent. Using this solution to test the method has another advantage: it is one of the testbeds for numerical codes suggested by the Apples with Apples alliance [1] and thus one may compare the results with other numerical methods. 
The plan of the article is as follows. In section 2 we shortly summarise the properties of discrete differential forms (more information can be found in $[6,10$, 28]). Then, in section 3 we describe the equations which result from a symmetry reduction. In section 4 we present the method of implementing these equations in two fully discrete numerical schemes. In section [5 we discuss how the schemes were tested, and in section 6 we present the results of those tests.

\section{Preliminaries}

Discrete differential forms in numerical GR were first introduced in [10]. The main feature of this method is its manifest coordinate independence. Coordinate invariance is one of the fundamental properties of GR. Thus, it is natural to use coordinate invariant numerical methods. A discretisation procedure with that property is Regge calculus $[13,27]$. However, so far it did not play a role in numerical GR. In computational approaches to treat the problem of coordinate dependencies multiple coordinate systems are used to cover the space-time $[14,30,31,35]$, but there the coordinate invariance is not manifest.

A fundamental difference of the discrete differential form approach in comparison with many other numerical methods is the discretisation of space-time. In contrast to the usual procedure, where the finite counterpart of the manifold is a numerical grid that is composed of a finite number of points (see e.g. [2] for a review article about numerical GR), the discrete space-time here also contains objects of higher dimensionality like curves and surfaces. The collection of points (nodes), curves (edges), surfaces (faces) and volumes is a cellular paving [6] and it is called computational mesh. The various elements of a cellular paving are called cells and through incidence relations between the cells the topology of the computational domain is defined. We use cellular pavings whose elements are all simplices, because this provides an elegant way to define a discrete version of the exterior product (see $[10,28]$ ). The cellular paving then corresponds to a simplicial complex [19].

Based on the cellular paving one discretises a theory that is formulated in terms of differential forms. Differential $p$-forms can be viewed as 'the objects which are integrated over $p$-dimensional submanifolds'. That means they provide maps from $p$-dimensional submanifolds to the reals. Then discrete $p$-forms are maps that assign a number to every $p$-dimensional cell in the cellular paving. They have received some attention since Bossavit [5] had pointed out that they correspond to the lowest order mixed finite element spaces defined by Nédélec [20] (see also [26]). Finite elements of mixed type have been used successfully in numerical applications to electrodynamics, see $[4,6,16]$. In numerical GR the finite element method has been used just recently e.g. in [32].

In order to use this approach one needs to have a formulation of geometries and, in particular, of GR which uses differential forms. A formulation of geometries based

on differential forms has been provided by É. Cartan [7]. The further step towards a formulation of GR using differential forms has been carried out by several authors. We mention here the work of Sparling [33] who has set up an exterior differential system of equations which is closed if and only if the vacuum Einstein equations hold. In [10] it is shown in detail how to set up the discrete formalism based on this exterior system using the ideas explained above.

In summary, the variables of our proposed discrete formulation will be the integrals of the differential forms in Sparling's formulation of GR over submanifolds. 
In order to get a finite number of variables we use a finite number of these submanifolds based on a triangulation of the computational domain. In this article we describe a simplification of the general formalism which occurs in space-times with a two-dimensional translational symmetry (see appendix A).

\section{The EFFECTIVE EQUATIONS}

To obtain the effective equations in the space-times of interest we start with the Cartan formulation of GR (using exterior forms) [33]. The basic variables in this formalism are the four 1 -forms of a pseudo-orthonormal tetrad $\boldsymbol{\theta}^{i}, i=0, \ldots, 3[18]$. With them the metric is

$$
g=\boldsymbol{\theta}^{0} \otimes \boldsymbol{\theta}^{0}-\boldsymbol{\theta}^{1} \otimes \boldsymbol{\theta}^{1}-\boldsymbol{\theta}^{2} \otimes \boldsymbol{\theta}^{2}-\boldsymbol{\theta}^{3} \otimes \boldsymbol{\theta}^{3}=\eta_{i k} \boldsymbol{\theta}^{i} \otimes \boldsymbol{\theta}^{k} .
$$

For the description of the connection in this formalism sixteen 1 -forms $\boldsymbol{\omega}^{i}{ }_{k}, i, k=$ $0, \ldots, 3$ are used. The connection should be compatible with the metric and torsion free, which translates to the antisymmetry-requirement and the first Cartanequation respectively]:

$$
\eta_{i k} \boldsymbol{\omega}^{k}{ }_{j}+\eta_{j k} \boldsymbol{\omega}^{k}{ }_{i}=0, \quad \mathbf{d} \boldsymbol{\theta}^{i}+\boldsymbol{\omega}^{i}{ }_{k} \boldsymbol{\theta}^{k}=0 .
$$

Furthermore, the metric should fulfil Einstein's vacuum field equations, which is equivalent to

$$
\mathbf{E}_{i}=0
$$

where $\mathbf{E}_{i}$ is the Einstein 3-form defined by (see [33])

$$
\mathbf{E}_{i}=\frac{1}{2} \varepsilon_{i j k l} \boldsymbol{\Omega}^{j k} \boldsymbol{\theta}^{l},
$$

with the curvature 2-form $\boldsymbol{\Omega}^{j}{ }_{k}=\mathbf{d} \boldsymbol{\omega}^{j}{ }_{k}+\boldsymbol{\omega}^{j}{ }_{l} \boldsymbol{\omega}^{l}{ }_{k}$ [9] .

Since the antisymmetry of the connection 1 -forms can easily be imposed, we are thus interested in the following system of equations

$$
\begin{aligned}
\mathbf{d} \boldsymbol{\theta}^{i}+\boldsymbol{\omega}^{i}{ }_{k} \boldsymbol{\theta}^{k} & =0, \\
\mathbf{E}_{i} & =0 .
\end{aligned}
$$

Sparling considered these equations on the frame bundle over the space-time manifold. He showed that Einstein's equations are satisfied if and only if the ideal generated by (5) is a closed differential ideal [33].

Even though the geometry is fixed by (5), there is still the freedom of choosing a gauge, i.e. there are Lorentz transformations $\Lambda^{i}{ }_{k}$ of the tetrad that do not change the metric

$$
g=\eta_{i k} \boldsymbol{\theta}^{i} \otimes \boldsymbol{\theta}^{k}=\eta_{i k}\left(\Lambda_{j}^{i} \boldsymbol{\theta}^{j}\right) \otimes\left(\Lambda^{k}{ }_{l} \boldsymbol{\theta}^{l}\right)=\left(\eta_{i k} \Lambda_{j}^{i} \Lambda_{l}^{k}\right) \boldsymbol{\theta}^{j} \otimes \boldsymbol{\theta}^{l} .
$$

In this work we will concentrate on general relativistic systems that occur in the context of space-times with a two-dimensional translational symmetry. Essentially that means that the group of translations $\mathbb{R}^{2}$ (or $\mathbb{T}^{2}$ ) acts isometrically on the space-time and that the orbits of this action are two-dimensional, space-like, flat submanifolds (the 'wave fronts'). The details are discussed in appendix $\mathrm{A}$ where it is also shown how to 'factor out' the symmetry action and how to derive an exterior system on the two-dimensional space $\mathcal{M}_{1}$ of orbits.

\footnotetext{
${ }^{1}$ Here and in what follows it is understood, that the product of differential forms is the antisymmetrised tensor-product, i.e. the exterior product.
} 
This procedure yields two exterior systems. The first one is

$$
\begin{aligned}
\mathbf{d}\left(f_{1}+g_{1}\right)-\left(f_{0}+g_{0}\right) \boldsymbol{\omega}+\left(f_{0} f_{1}+g_{0} g_{1}\right) \boldsymbol{\theta}^{0} & \\
+\left(f_{1}^{2}-f_{0} g_{0}+f_{1} g_{1}+g_{1}^{2}\right) \boldsymbol{\theta}^{1} & =0, \\
\mathbf{d}\left(f_{0}+g_{0}\right)-\left(f_{1}+g_{1}\right) \boldsymbol{\omega}+\left(f_{0} f_{1}+g_{0} g_{1}\right) \boldsymbol{\theta}^{1} & \\
+\left(f_{0}^{2}-f_{1} g_{1}+f_{0} g_{0}+g_{0}^{2}\right) \boldsymbol{\theta}^{0} & =0, \\
\mathbf{d} \boldsymbol{\omega}+\mathbf{d}\left(g_{0} \boldsymbol{\theta}^{1}+g_{1} \boldsymbol{\theta}^{0}\right)+\left(g_{0}^{2}-g_{1}^{2}\right) \boldsymbol{\theta}^{0} \boldsymbol{\theta}^{1} & =0 \\
\mathbf{d} \boldsymbol{\omega}+\mathbf{d}\left(f_{0} \boldsymbol{\theta}^{1}+f_{1} \boldsymbol{\theta}^{0}\right)+\left(f_{0}^{2}-f_{1}^{2}\right) \boldsymbol{\theta}^{0} \boldsymbol{\theta}^{1} & =0, \\
\mathbf{d} \boldsymbol{\theta}^{0}+\boldsymbol{\omega} \boldsymbol{\theta}^{1} & =0, \\
\mathbf{d} \boldsymbol{\theta}^{1}+\boldsymbol{\omega} \boldsymbol{\theta}^{0} & =0, \\
\mathbf{d}\left(f_{0} \boldsymbol{\theta}^{0}+f_{1} \boldsymbol{\theta}^{1}\right) & =0 .
\end{aligned}
$$

Here $\left(\boldsymbol{\theta}^{0}, \boldsymbol{\theta}^{1}\right)$ is a dyad in the two-dimensional orbit space $\mathcal{M}_{1}$ which carries a Lorentzian metric. The $S O(1,1)$ connection on this space is given by the 1 -form $\boldsymbol{\omega}^{0}{ }_{1}=: \boldsymbol{\omega}$. It is a consequence of the equations above that this connection is torsion free. The geometric properties of the orbits are described by the functions $f_{0}, f_{1}$, $g_{0}$ and $g_{1}$. For details see appendix A.

The other exterior system that we shall consider is obtained by a procedure analogous to the spherically symmetric case [28]. For the system (7) it is performed in [29]. One ends up with the following system

$$
\begin{aligned}
\mathbf{d}(\boldsymbol{\beta}+\boldsymbol{\delta})+(\boldsymbol{\alpha}+\boldsymbol{\gamma})(\boldsymbol{\beta}+\boldsymbol{\delta}) & =0, \\
\mathrm{~d} \boldsymbol{\omega}+\mathbf{d} \boldsymbol{\delta}+\boldsymbol{\gamma} \boldsymbol{\delta} & =0, \\
\mathrm{~d} \boldsymbol{\omega}+\mathbf{d} \boldsymbol{\beta}+\boldsymbol{\alpha} \boldsymbol{\beta} & =0, \\
\mathrm{~d} \boldsymbol{\theta}^{0}+\boldsymbol{\omega} \boldsymbol{\theta}^{1} & =0, \\
\mathbf{d} \boldsymbol{\theta}^{1}+\boldsymbol{\omega} \boldsymbol{\theta}^{0} & =0, \\
\mathbf{d} \boldsymbol{\alpha} & =0, \\
\mathbf{d} \boldsymbol{\gamma} & =0,
\end{aligned}
$$

where the 1-forms $\boldsymbol{\alpha}, \boldsymbol{\beta}, \boldsymbol{\gamma}$ and $\boldsymbol{\delta}$ are defined as

$$
\begin{aligned}
\boldsymbol{\alpha}:=f_{0} \boldsymbol{\theta}^{0}+f_{1} \boldsymbol{\theta}^{1}, & \boldsymbol{\beta}:=f_{1} \boldsymbol{\theta}^{0}+f_{0} \boldsymbol{\theta}^{1}, \\
\boldsymbol{\gamma}:=g_{0} \boldsymbol{\theta}^{0}+g_{1} \boldsymbol{\theta}^{1}, & \boldsymbol{\delta}:=g_{1} \boldsymbol{\theta}^{0}+g_{0} \boldsymbol{\theta}^{1} .
\end{aligned}
$$

To impose these correlation one uses the following algebraic equations

$$
\star \alpha=\beta, \quad \star \gamma=\delta,
$$

where $\star$ is the two-dimensional Hodge-operator [9].

The systems (77) and (8), (10) respectively now serve as the bases to derive numerical schemes. To achieve that we use the methods described in $[6,10,28]$.

\section{IMPLEMENTATION OF THE DisCRETE EQUATIONS}

In section 3 and appendix $\mathrm{A}$ we derived two systems of equations on the orbit space $\mathcal{M}_{1}$. Now we explain how these systems are discretised and develop numerical schemes. The first scheme is based on the system (7). It can be seen as the analogue 


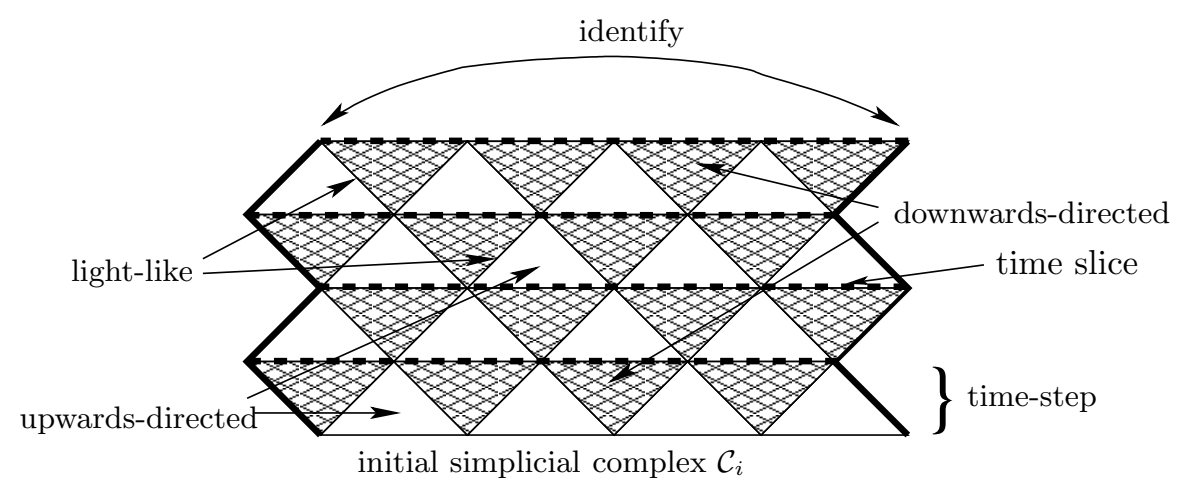

Figure 1: The triangulation $\mathcal{S}$ of the first four time-steps in the evolution of $\mathcal{C}_{i}$.

of scheme I in [28]. The second scheme is based on (8),(10) using the same ideas as scheme III in [28].

4.1. Properties of the simplicial mesh. The first step in the discretisation procedure is the definition of the finite counterpart of the manifold. In our case this step is common to both schemes. As we mentioned in section 2, that structure is a simplicial complex $\mathcal{S}$, and since we are in two dimensions that means it is composed of simplices of dimensions zero (nodes), one (edges) and two (faces).

Here the simplicial approximation $\mathcal{S}$ of a subset of the orbit space $\mathcal{M}_{1}$ is generated in analogy to the procedure described in [28]. Thus, the starting point for the construction of $\mathcal{S}$ is an initial hypersurface $\mathcal{I}$ and its discretisation, a 1-dimensional simplicial complex $\mathcal{C}_{i}$ [19], which we define so that it approximates a space-like curve in $\mathcal{M}_{1}$ (see section 5.2 for details). The difference here is that the topology of $\mathcal{I}$ is $S^{1}$, i.e. that we impose periodic boundary conditions. The domain of dependence of the initial hypersurface is thus the full orbit space $\mathcal{M}_{1}$.

Having obtained $\mathcal{C}_{i}$, a very elegant and invariant way to define the positions of the nodes in $\mathcal{S} \backslash \mathcal{C}_{i}$ is to send lightrays $l_{i}$ from the nodes in $\mathcal{C}_{i}$ and to take the intersections of these light-like geodesics as those positions.

By identifying links between these nodes with the edges of the simplicial complex appropriately one obtains the simplicial approximation $\mathcal{S}$. The construction is described in detail in [28] and its result is illustrated in figure 1.

The complex $\mathcal{S}$ contains two types of faces, the upwards and the downwards directed ones. In figure 1 the downwards directed faces are hatched, and the upwards directed ones are not. Moreover it contains two types of edges, namely space-like and light-like ones. Collecting the space-like edges one obtains a structure that, on the level of topology, appears as $N$ copies of the initial complex $\mathcal{C}_{i}$ (dashed lines in figure 1). Propagation from one of these copies (time-slice) to the next one is one time step.

A $p$-dimensional simplex is commonly denoted $\left[n_{0}, \ldots, n_{p}\right]$, where the $n_{i}$ are the corners of the simplex. In what follows we will denote the upwards and downwards directed faces $\left[n_{0}, n_{1}, n_{2}\right]$ and $\left[n_{0}^{\prime}, n_{1}^{\prime}, n_{2}^{\prime}\right]$ respectively, such that the edge $\left[n_{0}^{(\prime)}, n_{1}^{(\prime)}\right]$ is space-like and the edges $\left[n_{1}^{(\prime)}, n_{2}^{(\prime)}\right]$ as well as $\left[n_{0}^{(\prime)}, n_{2}^{(\prime)}\right]$ are light-like (see figure 2). 

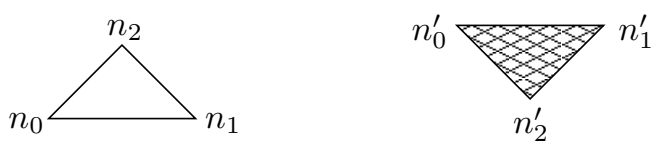

Figure 2: The upwards directed faces are denoted $\left[n_{0}, n_{1}, n_{2}\right]$ and the downwards directed faces are $\left[n_{0}^{\prime}, n_{1}^{\prime}, n_{2}^{\prime}\right]$.

As we already discussed in [28] the construction through light-like geodesics seems to be the simplest invariant method to define the position of $n_{2}$ in $(1+1)$ dimensional manifolds. The choice of the nodes at a later time and their connections to the nodes at the initial time is essentially arbitrary and only restricted by topological considerations. It is only when the $\boldsymbol{\theta}^{i}$ are known on all the edges that the geometry of the mesh is determined. We will see later that a part of these values can be specified freely while the rest is determined from the equations.

As in all numerical simulations degeneracies may occur. For instance two adjacent nodes in the same time slice may have a time-like distance. However, this must be seen as a sign that the mesh is too coarse and should be refined.

4.2. Properties of discrete forms. To discretise the equations (7) and (8) one needs discrete versions of the operations exterior product and exterior derivative. If the system (10) is taken into account additionally a discrete Hodge operator must be defined.

To get a discrete exterior product we use the method explained in [10] leading to the following formulas. Let $\boldsymbol{\alpha}^{p}$ and $\boldsymbol{\beta}^{q}$ be discrete $p$ - and $q$-forms respectively then

$$
\begin{aligned}
\boldsymbol{\alpha}^{0} \boldsymbol{\beta}^{q}\left[n_{0}, \ldots, n_{q}\right]= & \frac{1}{q+1}\left(\boldsymbol{\alpha}^{0}\left[n_{0}\right]+\ldots+\boldsymbol{\alpha}^{0}\left[n_{q}\right]\right) \boldsymbol{\beta}^{q}\left[n_{0}, \ldots, n_{q}\right], \\
\boldsymbol{\alpha}^{1} \boldsymbol{\beta}^{1}\left[n_{0}, n_{1}, n_{2}\right]=\frac{1}{6} & \left(\boldsymbol{\alpha}^{1}\left[n_{0}, n_{1}\right] \boldsymbol{\beta}^{1}\left[n_{0}, n_{2}\right]-\boldsymbol{\alpha}^{1}\left[n_{0}, n_{2}\right] \boldsymbol{\beta}^{1}\left[n_{0}, n_{1}\right]\right. \\
& +\boldsymbol{\alpha}^{1}\left[n_{1}, n_{2}\right] \boldsymbol{\beta}^{1}\left[n_{1}, n_{0}\right]-\boldsymbol{\alpha}^{1}\left[n_{1}, n_{0}\right] \boldsymbol{\beta}^{1}\left[n_{1}, n_{2}\right] \\
& \left.+\boldsymbol{\alpha}^{1}\left[n_{2}, n_{0}\right] \boldsymbol{\beta}^{1}\left[n_{2}, n_{1}\right]-\boldsymbol{\alpha}^{1}\left[n_{2}, n_{1}\right] \boldsymbol{\beta}^{1}\left[n_{2}, n_{0}\right]\right) .
\end{aligned}
$$

To obtain a natural discrete exterior derivative one applies Stokes' theorem. For us the relevant formulas are (see $[6,10])$

$$
\begin{aligned}
\mathbf{d} \boldsymbol{\alpha}^{0}\left[n_{0}, n_{1}\right] & =\boldsymbol{\alpha}^{0}\left[n_{1}\right]-\boldsymbol{\alpha}^{0}\left[n_{0}\right], \\
\mathbf{d} \boldsymbol{\alpha}^{1}\left[n_{0}, n_{1}, n_{2}\right] & =\boldsymbol{\alpha}^{1}\left[n_{1}, n_{2}\right]-\boldsymbol{\alpha}^{1}\left[n_{0}, n_{2}\right]+\boldsymbol{\alpha}^{1}\left[n_{0}, n_{1}\right] .
\end{aligned}
$$

The Hodge operator is discretised as described in the context of scheme III in [28]. This discretisation is based on the following observation. Let $\boldsymbol{\alpha}$ and $\boldsymbol{\beta}$ be 1-forms such that $\boldsymbol{\alpha}=\star \boldsymbol{\beta}$. When this equation is integrated over a light-like geodesic $\gamma_{\varepsilon}$ with $\int_{\gamma_{\varepsilon}} \boldsymbol{\theta}^{0}=\varepsilon \int_{\gamma_{\varepsilon}} \boldsymbol{\theta}^{1}(\varepsilon \in\{-1,+1\})$ then also

$$
\int_{\gamma_{\varepsilon}} \boldsymbol{\alpha}=\varepsilon \int_{\gamma_{\varepsilon}} \boldsymbol{\beta}
$$


Thus we use the following discretisation of the Hodge operator. When the edge $\left[n_{1}, n_{2}\right]$ is light-like then

$$
\star \boldsymbol{\beta}\left[n_{1}, n_{2}\right]=\varepsilon \boldsymbol{\alpha}\left[n_{1}, n_{2}\right] .
$$

In (10) the Hodge operator is only applied to 1 -forms. Therefore it is not necessary to define its discrete version for general forms here.

Having a simplicial mesh and the discrete operators we can now develop the numerical schemes. Common to both schemes is that for each triangle a system of equations has to be solved. These are coupled non-linear algebraic equations. Their analysis is somewhat complicated and their properties are not yet clear. They might not have a unique solution. However, at least one solution can be found by Newton's iteration method. We used the GNU Scientific Library, especially the implementation of the modified Powell method [11,24].

4.3. Scheme I. The first scheme is based on the system (7). The variables are the discrete 1 -forms $\boldsymbol{\theta}^{0}, \boldsymbol{\theta}^{1}$ and $\boldsymbol{\omega}$ as well as the discrete 0 -forms $f_{0}, f_{1}, g_{0}$ and $g_{1}$. For the upwards directed faces the numbers

$$
\begin{aligned}
& \left\{f_{0}\left[n_{0}\right], f_{0}\left[n_{1}\right], f_{1}\left[n_{0}\right], f_{1}\left[n_{1}\right], g_{0}\left[n_{0}\right], g_{0}\left[n_{1}\right], g_{1}\left[n_{0}\right], g_{1}\left[n_{1}\right],\right. \\
& \left.\boldsymbol{\theta}^{0}\left[n_{0}, n_{1}\right], \boldsymbol{\theta}^{1}\left[n_{0}, n_{1}\right], \boldsymbol{\omega}\left[n_{0}, n_{1}\right]\right\}
\end{aligned}
$$

are given initial data, and

$$
\begin{aligned}
& \left\{f_{0}\left[n_{2}\right], f_{1}\left[n_{2}\right], g_{0}\left[n_{2}\right], g_{1}\left[n_{2}\right],\right. \\
& \left.\quad \boldsymbol{\theta}^{0}\left[n_{0}, n_{2}\right], \boldsymbol{\theta}^{0}\left[n_{1}, n_{2}\right], \boldsymbol{\theta}^{1}\left[n_{0}, n_{2}\right], \boldsymbol{\theta}^{1}\left[n_{1}, n_{2}\right], \boldsymbol{\omega}\left[n_{0}, n_{2}\right], \boldsymbol{\omega}\left[n_{1}, n_{2}\right]\right\}
\end{aligned}
$$

are the unknowns.

The system (7) is composed of two 1-form equations (7a), (7b) and five 2-form equations (7c)-(7g). Therefore, since there are two 1-form equations on each of the light-like edges and five 2-form equations on the upwards directed face, it is clear that the total number of equations in the discretised system is nine. However, we discuss in appendix $\mathrm{A}$ that on the continuous manifold the 1-form equations (7a), (7b) take a special role, because they are not independent of the remaining equations in (7). They are partially redundant. Therefore we require the discrete 1 -form equations (7a), (7b) to be satisfied at the edge $\left[n_{0}, n_{2}\right]$, but we do not require these equations at the edge $\left[n_{1}, n_{2}\right]$.

Hence, we have seven equations for the upwards directed faces and ten unknowns. We get three more equations by using the definition of the position of $n_{2}$ and fixing the gauge outside the initial hypersurface. That means we require

$$
\left(\boldsymbol{\theta}^{0}-\boldsymbol{\theta}^{1}\right)\left[n_{0}, n_{2}\right]=0, \quad \quad\left(\boldsymbol{\theta}^{0}+\boldsymbol{\theta}^{1}\right)\left[n_{1}, n_{2}\right]=0
$$

to specify the position of $n_{2}$, and the gauge is chosen such that

$$
\boldsymbol{\omega}\left[n_{0}, n_{2}\right]+\boldsymbol{\omega}\left[n_{1}, n_{2}\right]=0 .
$$

This gauge choice was also used in scheme III of [28].

For the downwards directed faces the unknowns are

$$
\left\{\boldsymbol{\theta}^{0}\left[n_{0}^{\prime}, n_{1}^{\prime}\right], \boldsymbol{\theta}^{1}\left[n_{0}^{\prime}, n_{1}^{\prime}\right], \boldsymbol{\omega}\left[n_{0}^{\prime}, n_{1}^{\prime}\right]\right\} .
$$

We already discussed how the observation, that the equations (7a), (7b) are redundant, can be implemented on the discrete level. Following that reasoning we conclude that those 1 -form equations need not be required at $\left[n_{0}^{\prime}, n_{1}^{\prime}\right]$. 
It follows that we only need to consider the system (7c)- (7g). These are five 2 -form equations, but the number of unknowns is only three. Thus, the system is overdetermined and we have to select three of the five equations $(7 \mathrm{c})-(7 \mathrm{~g})$. At the moment it is not clear how a reasonable choice should be made. Compared to other choices that were tested shortly and provided rapidly growing errors the following system seems to work quite well

$$
\begin{aligned}
& 0=\mathbf{d} \boldsymbol{\theta}^{0}+\boldsymbol{\omega} \boldsymbol{\theta}^{1}, \\
& 0=\mathbf{d} \boldsymbol{\theta}^{1}+\boldsymbol{\omega} \boldsymbol{\theta}^{0}, \\
& 0=\mathbf{d} \boldsymbol{\omega}+\mathbf{d}\left(g_{1} \boldsymbol{\theta}^{0}+g_{0} \boldsymbol{\theta}^{1}\right)+\left(g_{0}^{2}-g_{1}^{2}\right) \boldsymbol{\theta}^{0} \boldsymbol{\theta}^{1} .
\end{aligned}
$$

Altogether these are seven equations and seven unknowns for the upwards directed faces, as well as three equations and three unknowns for the downwards directed ones.

4.4. Scheme II. To obtain the second scheme we discretise the equations (8a)(8g), (10), i.e.

$$
\begin{array}{ll}
0=\mathbf{d} \boldsymbol{\theta}^{0}+\boldsymbol{\omega} \boldsymbol{\theta}^{1}, & 0=\mathbf{d} \boldsymbol{\theta}^{1}+\boldsymbol{\omega} \boldsymbol{\theta}^{0}, \\
0=\mathbf{d} \boldsymbol{\omega}+\mathbf{d} \boldsymbol{\delta}+\boldsymbol{\gamma} \boldsymbol{\delta}, & 0=\mathbf{d} \boldsymbol{\omega}+\mathbf{d} \boldsymbol{\beta}+\boldsymbol{\alpha} \boldsymbol{\beta}, \\
0=\mathbf{d} \boldsymbol{\gamma}, & 0=\mathbf{d} \boldsymbol{\alpha}, \\
0=\mathbf{d}(\boldsymbol{\beta}+\boldsymbol{\delta})+(\boldsymbol{\alpha}+\gamma)(\boldsymbol{\beta}+\boldsymbol{\delta}), & \\
0=\star \boldsymbol{\beta}-\boldsymbol{\alpha}, & 0=\star \boldsymbol{\delta}-\boldsymbol{\gamma} .
\end{array}
$$

That means the variables are the seven 1 -forms $\boldsymbol{\alpha}, \boldsymbol{\beta}, \boldsymbol{\gamma}, \boldsymbol{\delta}, \boldsymbol{\theta}^{0}, \boldsymbol{\theta}^{1}$ and $\boldsymbol{\omega}$. The given initial data are hence

$$
\begin{gathered}
\left\{\boldsymbol{\alpha}\left[n_{0}, n_{1}\right], \boldsymbol{\beta}\left[n_{0}, n_{1}\right], \boldsymbol{\gamma}\left[n_{0}, n_{1}\right], \boldsymbol{\delta}\left[n_{0}, n_{1}\right],\right. \\
\left.\boldsymbol{\theta}^{0}\left[n_{0}, n_{1}\right], \boldsymbol{\theta}^{1}\left[n_{0}, n_{1}\right], \boldsymbol{\omega}\left[n_{0}, n_{1}\right]\right\},
\end{gathered}
$$

and the unknowns for the upwards directed faces are

$$
\begin{aligned}
\left\{\boldsymbol{\alpha}\left[n_{0}, n_{2}\right], \boldsymbol{\alpha}\left[n_{1}, n_{2}\right], \boldsymbol{\beta}\left[n_{0}, n_{2}\right], \boldsymbol{\beta}\left[n_{1}, n_{2}\right],\right. \\
\quad \gamma\left[n_{0}, n_{2}\right], \boldsymbol{\gamma}\left[n_{1}, n_{2}\right], \boldsymbol{\delta}\left[n_{0}, n_{2}\right], \boldsymbol{\delta}\left[n_{1}, n_{2}\right], \\
\left.\quad \boldsymbol{\theta}^{0}\left[n_{0}, n_{2}\right], \boldsymbol{\theta}^{0}\left[n_{1}, n_{2}\right], \boldsymbol{\theta}^{1}\left[n_{0}, n_{2}\right], \boldsymbol{\theta}^{1}\left[n_{1}, n_{2}\right], \boldsymbol{\omega}\left[n_{0}, n_{2}\right], \boldsymbol{\omega}\left[n_{1}, n_{2}\right]\right\} .
\end{aligned}
$$

The system (21) is composed of seven 2 -form equations and two algebraic equations involving the Hodge operator. These equations are discretised with the exterior product (11), the exterior derivative (12) and the Hodge operator (14). For the upwards-directed faces this procedure results in seven equations for every face and two equations for both of the two light-like edges. In total these are eleven equations.

With the same procedure as in scheme I, i.e. using that the new edges are lightlike and choosing a gauge with $\boldsymbol{\omega}\left[n_{0}, n_{2}\right]+\boldsymbol{\omega}\left[n_{1}, n_{2}\right]=0$, we reduce the number of unknowns from fourteen to eleven, such that the number of equations for the upwards directed faces equals the number of unknowns there.

For the downwards directed faces the unknowns are the integrals of the seven 1 -forms along the space-like edge, i.e. we have seven unknowns

$$
\begin{gathered}
\left\{\boldsymbol{\alpha}\left[n_{0}^{\prime}, n_{1}^{\prime}\right], \boldsymbol{\beta}\left[n_{0}^{\prime}, n_{1}^{\prime}\right], \boldsymbol{\gamma}\left[n_{0}^{\prime}, n_{1}^{\prime}\right], \boldsymbol{\delta}\left[n_{0}^{\prime}, n_{1}^{\prime}\right],\right. \\
\left.\boldsymbol{\theta}^{0}\left[n_{0}^{\prime}, n_{1}^{\prime}\right], \boldsymbol{\theta}^{1}\left[n_{0}^{\prime}, n_{1}^{\prime}\right], \boldsymbol{\omega}\left[n_{0}^{\prime}, n_{1}^{\prime}\right]\right\} .
\end{gathered}
$$


The discrete equations involving the Hodge operator are already satisfied at the light-like edges $\left[n_{0}^{\prime}, n_{2}^{\prime}\right]$ and $\left[n_{1}^{\prime}, n_{2}^{\prime}\right]$. Hence we get seven discrete equations from the 2 -forms in (21), i.e. from (8a)- (8g).

\section{TEST-SCEnARIOS}

In the last chapters we described, how discrete differential forms can be applied to study systems with a planar symmetry in GR. In this chapter we want to present the concrete example, where the code was tested. The idea for the test is to derive discrete initial data from an analytical solution, use the numerical schemes to simulate the time evolution and finally compare the numerical results with the analytically expected ones. That means an analytical solution is needed.

As a testbed we have chosen the polarised Gowdy space-time $[1,15]$, because it has at least three advantages. Its spatial slices have the topology $\mathbb{T}^{3}$, the light-like geodesics take a very simple form and it is suggested as a testbed for numerical schemes by the Apples with Apples alliance [1]. In standard coordinates $\{t, z, x, y\}$ its metric reads

$$
g=t^{-1 / 2} e^{\lambda / 2}(\mathbf{d} t \otimes \mathbf{d} t-\mathbf{d} z \otimes \mathbf{d} z)-t e^{P} \mathbf{d} x \otimes \mathbf{d} x-t e^{-P} \mathbf{d} y \otimes \mathbf{d} y,
$$

where $t \in \mathbb{R}, z \in[0,1]$,

$$
\begin{aligned}
P(t, z)= & J_{0}(2 \pi t) \cos 2 \pi z, \\
\lambda(t, z)=- & 2 \pi t J_{0}(2 \pi t) J_{1}(2 \pi t) \cos ^{2} 2 \pi z+2 \pi^{2} t^{2}\left[J_{0}^{2}(2 \pi t)+J_{1}^{2}(2 \pi t)\right] \\
& -2 \pi^{2}\left[J_{0}^{2}(2 \pi)+J_{1}^{2}(2 \pi)\right]+\pi J_{0}(2 \pi) J_{1}(2 \pi),
\end{aligned}
$$

and $J_{0}, J_{1}$ are the usual Bessel-functions.

It can easily be checked that a light-like geodesic in the $(t, z)$-surface through a point with coordinates $\left(t_{0}, z_{0}\right)$ also contains the points $\left(t_{0}+k, z_{0}\right)(k \in \mathbb{N})$. In this sense, a massles test particle needs a time of $\delta t=1$ to travel around the universe. This time interval is commonly denoted a crossing time.

5.1. The continuous forms. To get the differential forms we make a gauge-choice, i.e. we choose some $\boldsymbol{\theta}^{0}$ and $\boldsymbol{\theta}^{1}$, that generate the corresponding metric. In polarised Gowdy geometry a natural choice leads to

$$
\begin{aligned}
f_{0} & =\frac{1}{2} t^{\frac{1}{4}} e^{-\frac{\lambda}{4}}\left(\frac{1}{t}+\partial_{t} P\right), & f_{1} & =\frac{1}{2} t^{\frac{1}{4}} e^{-\frac{\lambda}{4}} \partial_{z} P, \\
g_{0} & =\frac{1}{2} t^{\frac{1}{4}} e^{-\frac{\lambda}{4}}\left(\frac{1}{t}-\partial_{t} P\right), & g_{1} & =-\frac{1}{2} t^{\frac{1}{4}} e^{-\frac{\lambda}{4}} \partial_{z} P, \\
\boldsymbol{\theta}^{0} & =t^{-1 / 4} e^{\lambda / 4} d t, & \boldsymbol{\theta}^{1} & =t^{-1 / 4} e^{\lambda / 4} d z, \\
\boldsymbol{\alpha} & =\frac{1}{2}\left(\partial_{z} P d z+\partial_{t} P d t+\frac{1}{t} d t\right), & \boldsymbol{\beta} & =\frac{1}{2}\left(\partial_{z} P d t+\partial_{t} P d z+\frac{1}{t} d z\right), \\
\boldsymbol{\gamma} & =\frac{1}{2}\left(\frac{1}{t} d t-\partial_{t} P d t-\partial_{z} P d z\right), & \boldsymbol{\delta} & =\frac{1}{2}\left(\frac{1}{t} d z-\partial_{t} P d z-\partial_{z} P d t\right), \\
\boldsymbol{\omega} & =\frac{1}{4}\left(\partial_{z} \lambda d t+\partial_{t} \lambda d z-\frac{1}{t} d z\right), & &
\end{aligned}
$$


where for the derivatives of $P$ and $\lambda$ we get

$$
\begin{aligned}
\partial_{t} P(t, z) & =-2 \pi J_{1}(2 \pi t) \cos 2 \pi z, \\
\partial_{z} P(t, z) & =-2 \pi J_{0}(2 \pi t) \sin 2 \pi z, \\
\partial_{t} \lambda(t, z) & =t\left(\left(\partial_{t} P(t, z)\right)^{2}+\left(\partial_{z} P(t, z)\right)^{2}\right), \\
\partial_{z} \lambda(t, z) & =2 t\left(\partial_{t} P(t, z)\right)\left(\partial_{z} P(t, z)\right) .
\end{aligned}
$$

5.2. Association with discrete forms. The next step is to choose an initial hypersurface. This has to obey our requirement that its topology is $S^{1}$. That means we need a closed space-like curve in the two-dimensional orbit space $\mathcal{M}_{1}$ 2 For the test we used curves, whose $t$-coordinate is constant

$$
\left(\begin{array}{c}
t \\
z
\end{array}\right)=\left(\begin{array}{c}
t_{0} \\
z_{0}
\end{array}\right)+\lambda\left(\begin{array}{l}
0 \\
1
\end{array}\right)
$$

with $\lambda \in[0,1)$ and fixed $t_{0}, z_{0}$.

We subdivide this curve into $n$ pieces, which are again of the form (28), but with $\lambda \in[(i-1) / n, i / n], i=1, \ldots, n$. On each of these pieces we integrate the 1 -forms, and on the nodes we evaluate the 0 -forms to get initial values.

Since light-rays in the polarized Gowdy solution (25) are the curves with $|d t|=$ $|d z|$, the coordinate of the nodes in the computational mesh are easily obtained and it is thus straight forward to compare the numerical and the analytical solution (see [28]).

This is done as follows. In every time slice there are $n>1$ space-like edges. We take the maximum of the relative error of the invariant length 3 of the edges in a time slice as a measure for the accuracy of the scheme.

In this construction, since $n$ is the number of initial edges, we need $2 n$ time steps to simulate a crossing time $(\delta t=1)$.

\section{Concrete Examples And Results}

Now two scenarios in the polarised Gowdy solution are investigated, an expanding and a collapsing time evolution. For the expanding evolution we take an initial hypersurface of the form (28) with $t_{0}=t_{0}^{e}:=1$ and $z_{0}=z_{0}^{e}:=0$, and for the collapsing evolution these constants are $t_{0}=t_{0}^{c}:=9.8753205829098$ and $z_{0}=z_{0}^{c}:=-0.5$.

The hypersurface $t_{0}=t_{0}^{c}$ is in the sense special that the Bessel function term $J_{0}(2 \pi t)$ vanishes and the Apples with Apples alliance suggests to use this particular choice.

6.1. Expanding Evolution. In the expanding evolution we divide the initial curve into $n$ edges of the form $\{z=\lambda, t=1, \lambda \in[(k-1) / n, k / n], k=1, \ldots, n\}$. For $n=50$ and $n=100$ the growth in time of the maximal relative error of the lengths of space-like edges is shown in figure 3 .

For scheme II we see that, although the absolute value of the lengths of the edges grows exponentially in this space-time, the relative error of these lengths grows only linearly and remains small even at $t=100$. Furthermore in this scheme the error in the calculation with 100 initial edges is about 4 times smaller than the error with 50 initial edges. This indicates quadratic convergence for decreasing edge lengths.

\footnotetext{
${ }^{2}$ The orbit space $\mathcal{M}_{1}$ can be identified with a surface that satisfies $x=$ const, $y=$ const.

${ }^{3}$ We define the relative error to be $\left|l_{a p} / l_{e x}-1\right|$, where $l_{a p}$ is the numerically calculated approximation to the invariant length and $l_{e x}$ is the length in the exact solution.
} 

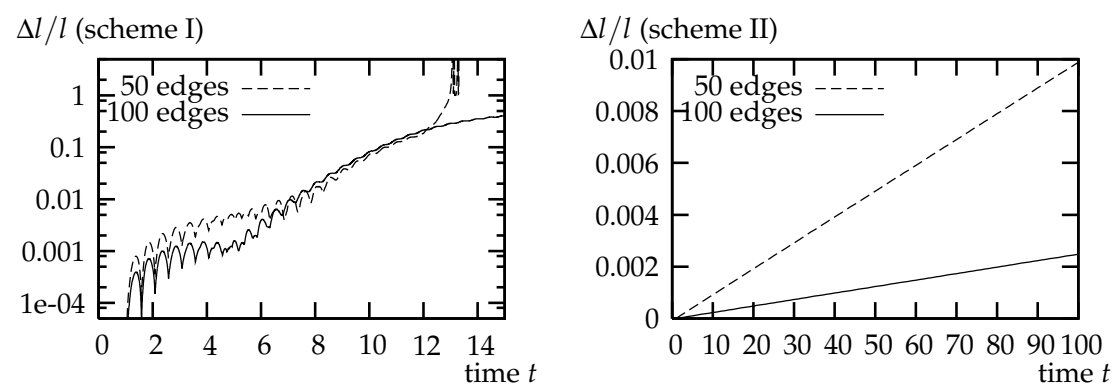

Figure 3: Expanding evolution: Growth of the maximal relative error of the invariant lengths of the space-like edges. Left: Results of scheme I (logarithmic ordinate). Right: Results of scheme II (linear ordinate).

For scheme I the error does not grow only linearly. We see that for three to four crossing times the scheme has a good numerical behaviour. The size of the error grows slowly, it is relatively small and becomes even smaller when more initial edges are used. But after four crossing times the error starts to grow exponentially, and the results of the calculations in different meshes become similar. Then, at $t \approx 12$ the error in the simulation with 50 edges blows up rapidly. Similar behaviour can be observed for the simulation with 100 initial edges, but here the critical time is $t \approx 20$.

In both schemes the growing of the error is superposed by a quasi-periodical fluctuation (for scheme II it is not visible in figure 3. because it is small, but it exists). The period of this fluctuation is $\delta t=1 / 2$, i.e. half as long as the crossing time.

6.2. Collapsing Evolution. For the collapsing evolution we again divide the initial curve into $n$ edges of the form $\left\{z=\lambda-0.5, t=t_{0}^{c}, \lambda \in[(k-1) / n, k / n], k=\right.$ $1, \ldots, n\}$. For $n=50$ and $n=100$ the growth of the maximal relative error of the lengths of space-like edges is shown in figure 4

Since at $t=0$ the polarised Gowdy space-time has a singularity (the cosmological singularity), we can only evolve as long as $t$ remains positive. I.e. if we use for instance 50 initial edges, we can at most make 987 time-steps.

A surprising result is that for both schemes the relative error does not grow at all. Instead it fluctuates quasi-periodically, but remains at similar sizes until the time approaches zero, i.e. near the singularity.

Here the period of the fluctuation is $\delta t=1 / 4$, i.e. a quarter of a crossing time and only half as long as in the expanding time evolution. Further investigations reveal that for other initial hypersurfaces, i.e. $t_{0} \neq t_{0}^{c}$, the error still fluctuates, but then the period of the fluctuation is $\delta t=1 / 2$, like in the expanding time evolution.

Another point to mention is that for scheme II the error in the simulation with 100 edges is about four times smaller than the error in the simulation with 50 edges. This is not the case in scheme I where the errors of both simulations are of comparable size.

6.3. Convergence of the Schemes. Finally we consider the convergence behaviour of the numerical schemes for both examples. We saw that in the expanding 

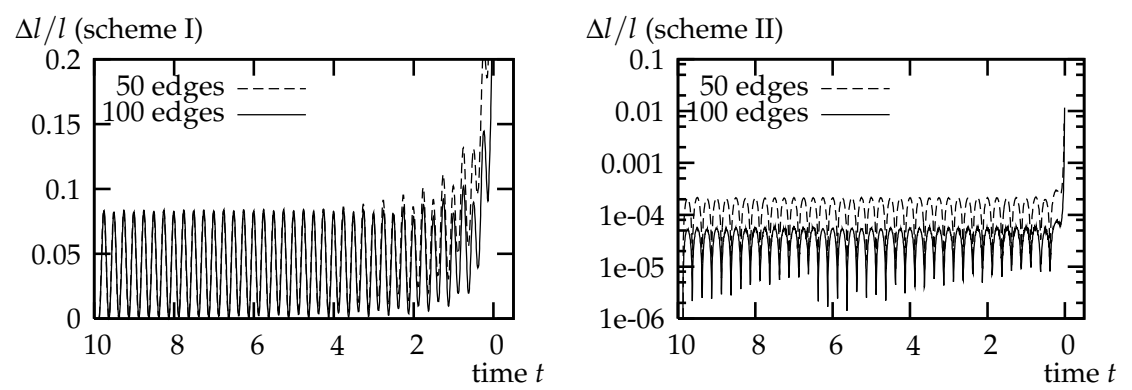

Figure 4: Collapsing evolution: Maximal relative error of the invariant lengths of the space-like edges. Left: Results of scheme I. Right: Results of scheme II.
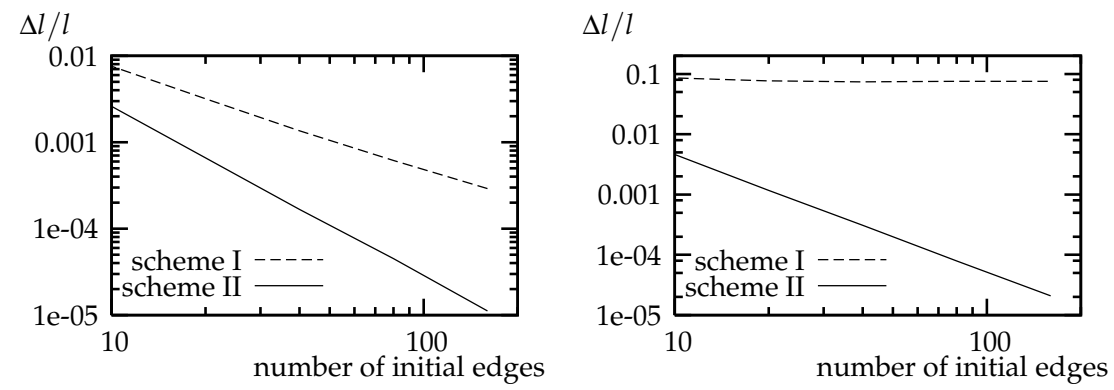

Figure 5: Convergence of the schemes. Left: expanding evolution at $t=2$. Right: collapsing evolution at $t=t_{0}^{c}-4.9$.

time evolution scheme I is not stable, and that it behaves best for $t<4$. Therefore we investigate the errors at $t=2$. For the collapsing evolution we choose with $t=t_{0}^{c}-4.9$ a time where the (fluctuating) error has neither a minimum nor a maximum.

In figure 5 it is shown how both schemes behave when the typical length of the edges is decreased, i.e. when the number of initial edges becomes larger. To obtain the curves we calculated the time evolution with 10, 20, 40, 80 and 160 initial edges.

We see that the results of scheme II converge quadratically to the analytical solution. The errors of scheme I on the other hand converge only linearly to zero in the expanding time evolution and do not converge at all in the collapsing time evolution. This is in agreement with the observations that we made in the time evolution simulations.

6.4. Discussion. The first point to discuss is the quasi-periodical fluctuation of the error and its period of $\delta t=1 / 2$. This phenomenon points to the interpretation that, due to the periodic boundary conditions, the errors that sum up during the time evolution cancel out when a lightray intersects the other lightray that was sent from the same point in the other direction.

In the collapsing time evolution we observed that the period of the fluctuation is only $\delta t=1 / 4$ when $t_{0}=t_{0}^{c}$, but it is again $\delta t=1 / 2$ when other initial data are 
chosen. This initial hypersurface is in the sense special that the Bessel function $J_{0}(2 \pi t)$ has a root there. Hence it is most likely that with these initial data other symmetries of the considered space-time cause new cancellations of errors.

Concerning the quality of the numerical solutions we see that (at least for the considered examples) scheme II provides the best results. This scheme is accurate and stable, the relative error grows only linearly in time and remains small. Unfortunately we cannot say much about its robustness (in the sense of the Apples with Apples alliance), because we only considered the polarised Gowdy space-time. This scheme is the analogue of scheme III in [28], which also provided the best results there. The reason for this behaviour seems to be that the system (8) is geometrically preferred.

The analogue of scheme I here is scheme I in [28]. For spherically symmetric space-times the results of that scheme were, compared to the results of scheme I here, better. Especially it was quadratically convergent.

For scheme I here there are situations when it does not converge at all, and moreover it is not stable. We saw that for small times in the expanding evolution it behaves quite well, but after that there is a period when the error grows exponentially. Up to now it is not clear if the reason for this behaviour is rather the numerical scheme or the exponentially growing example. However, it becomes clear that the scheme is unstable when the period of exponential growth ends (at $t \approx 12$ resp. $t \approx 20)$. We thus conclude that scheme I is not practicable.

Of course it is not too surprising that problems occur in scheme I, because the system (7) is not very well understood at the analytical level. In particular, we were not able to derive a minimal system for the gravitational wave space-times. Quite the contrary we found an overdetermined system, and although there are arguments that some equations are redundant, it was still necessary to choose discrete equations that we omitted without having justifying arguments for this particular choice.

Comparing the previously presented numerical schemes with other codes one has to distinguish between the expanding and the collapsing case. The reason is that in the collapsing time evolution the coordinate representation (25) is usually not used. Instead one takes a harmonic time coordinate $\tau$ that becomes infinite at the singularity [25]. That means that even if the difference of $\tau$ between the space-like slices is fixed, the difference of $t$ becomes small. Yet, because of our method to construct the computational mesh we cannot control the slicing in this way. The results of other numerical methods are thus not really comparable.

However, simulations of collapsing polarised Gowdy evolutions can be found e.g. in $[12,25]$. Furthermore [1] reports about results of the ADM and BSSN codes and the PITT group [23] presents results of the ABIGEL code. Further information about cosmological space-times with singularities can be found e.g. in [3].

Numerical simulations that use the expanding polarised Gowdy solution as a testbed can be found e.g. in [21]. Again the PITT group [23] presents results of the ABIGEL code.

Summarising the hitherto existing work about discrete differential forms in numerical GR it seems that the most urgent task, in order to apply this method to physically more relevant situations, is to find statements about the underlying system of equations. Indeed it is not clear how to apply standard concepts of numerical 
GR, like hyperbolicity, to the Cartan formulation. What is currently lacking is a better understanding of the notions of a discrete geometry.

\section{ACKNOWLEDGEMENTS}

The authors are grateful to A Bossavit for helpful discussions and suggestions. This work is supported by the SFB 382 project on "Methods and algorithms for the simulation of physical processes on high performance computers".

\section{Appendix A. Derivation of the Reduced System}

We are interested in space-times $(\mathcal{M}, g)$ which can be characterised by the following conditions:

(1) There is an effective, isometric action of $\mathbb{R}^{2}$ on $\mathcal{M}$.

(2) The generators of this action, the two Killing vectors $\xi$ and $\eta$, are space-like, they are orthogonal and they commute.

(3) The action is hypersurface-orthogonal, i.e., the 2-flats orthogonal to the span of the Killing vectors are integrable.

From condition (1) it follows that the space-time is locally foliated by 2-dimensional orbits whose tangent spaces are spanned by the Killing vectors. Since these are orthogonal and space-like we can define two space-like unit vectors by

$$
\xi=\mathrm{e}^{f} e_{2}, \quad \eta=\mathrm{e}^{g} e_{3},
$$

with functions $f$ and $g$ which are constant on the orbits. We complete these vectors to an orthonormal basis $\left(e_{0}, e_{1}, e_{2}, e_{3}\right)$ with dual basis $\left(\boldsymbol{\theta}^{0}, \boldsymbol{\theta}^{1}, \boldsymbol{\theta}^{2}, \boldsymbol{\theta}^{3}\right)$. Since $\left[e_{2}, e_{3}\right]=0$ we have

$$
\mathbf{d} \boldsymbol{\theta}^{0}\left(e_{2}, e_{3}\right)=e_{2}\left(\boldsymbol{\theta}^{0}\left(e_{3}\right)\right)-e_{3}\left(\boldsymbol{\theta}^{0}\left(e_{2}\right)\right)-\boldsymbol{\theta}^{0}\left(\left[e_{2}, e_{3}\right]\right)=0
$$

and, similarly, for $\boldsymbol{\theta}^{1}$. Hence, we get the expansions

$$
\mathbf{d} \boldsymbol{\theta}^{0}=\mathbf{A} \boldsymbol{\theta}^{0}+\mathbf{B} \boldsymbol{\theta}^{1}, \quad \mathbf{d} \boldsymbol{\theta}^{1}=\mathbf{C} \boldsymbol{\theta}^{0}+\mathbf{D} \boldsymbol{\theta}^{1}
$$

for some 1-forms $\mathbf{A}, \mathbf{B}, \mathbf{C}$ and $\mathbf{D}$. This can be expressed by the well known integrability conditions

$$
\mathbf{d} \boldsymbol{\theta}^{0} \boldsymbol{\theta}^{0} \boldsymbol{\theta}^{1}=0, \quad \mathbf{d} \boldsymbol{\theta}^{1} \boldsymbol{\theta}^{0} \boldsymbol{\theta}^{1}=0 .
$$

While the choice of $e_{2}$ and $e_{3}$ is fixed by aligning them with the Killing vectors the other two basis vectors are not fixed. They can still rotate inside the plane which they generate with a rotation which may not only depend on the orbit but even the location on the orbit. We can fix the latter dependence by requiring that $e_{0}$ and $e_{1}$ commute with both Killing vectors. This requirement is consistent because the Killing vectors commute.

Then the four basis vectors are Lie transported along both Killing vectors and the same is true for the dual basis vectors, i.e., the equations

$$
\mathcal{L}_{\xi} \boldsymbol{\theta}^{a}=0=\mathcal{L}_{\eta} \boldsymbol{\theta}^{a}
$$

hold for $a=0, \ldots, 3$. Using these equations and the expansions (31) leads us after a short calculation to the fact that the 1 -forms $\mathbf{A}$ to $\mathbf{D}$ are linear combinations of $\boldsymbol{\theta}^{0}$ and $\boldsymbol{\theta}^{1}$ only, i.e., they vanish when restricted to an orbit. 
Furthermore, condition (3) implies that the distribution generated by $e_{0}$ and $e_{1}$ is integrable, i.e., that

$$
\left[e_{0}, e_{1}\right]=\alpha e_{0}+\beta e_{1} .
$$

This implies that there exist 2-dimensional submanifolds orthogonal to the orbits of the action which are dragged into each other by the group action.

Expressed in terms of the dual basis, the integrability condition is

$$
\mathbf{d} \boldsymbol{\theta}^{2} \boldsymbol{\theta}^{2} \boldsymbol{\theta}^{3}=0, \quad \mathbf{d} \boldsymbol{\theta}^{3} \boldsymbol{\theta}^{2} \boldsymbol{\theta}^{3}=0,
$$

which leads to a similar expansion of the differentials in terms of the coframe as above. In an analogous calculation making use of the invariance of the frame (33) we find the following relations

$$
\mathbf{d} \boldsymbol{\theta}^{2}=\mathbf{d} f \boldsymbol{\theta}^{2}, \quad \mathbf{d} \boldsymbol{\theta}^{3}=\mathbf{d} g \boldsymbol{\theta}^{3} .
$$

These relationships allow us to partly determine the connection forms from the first structure equation

$$
\mathbf{d} \boldsymbol{\theta}^{a}+\boldsymbol{\omega}_{b}^{a} \boldsymbol{\theta}^{b}=0 .
$$

We find that all the connection forms except for $\boldsymbol{\omega}:=\boldsymbol{\omega}^{0}{ }_{1}$ are determined from the above equations

$$
\boldsymbol{\omega}_{0}^{2}=f_{0} \boldsymbol{\theta}^{2}, \quad \boldsymbol{\omega}^{2}{ }_{1}=f_{1} \boldsymbol{\theta}^{2}, \quad \boldsymbol{\omega}^{3}{ }_{0}=g_{0} \boldsymbol{\theta}^{3}, \quad \boldsymbol{\omega}^{3}{ }_{1}=g_{1} \boldsymbol{\theta}^{3}, \quad \boldsymbol{\omega}^{2}{ }_{3}=0,
$$

where $f_{0}=e_{0}(f)$, etc. so that $\mathbf{d} f=f_{0} \boldsymbol{\theta}^{0}+f_{1} \boldsymbol{\theta}^{1}$. So we find that the information about the extrinsic geometry of the orbits is contained in the two scalars $f$ and $g$, while the geometry of the orbit space $\mathcal{M}_{1}$ which can be identified with the space orthogonal to the orbits is described by the coframe vectors $\left(\boldsymbol{\theta}^{0}, \boldsymbol{\theta}^{1}\right)$ and the connection form $\boldsymbol{\omega}$.

With these expressions for the connection forms we get the Nester-Witten 2forms

$$
\begin{array}{ll}
L_{0}=\left(f_{1}+g_{1}\right) \boldsymbol{\theta}^{2} \boldsymbol{\theta}^{3}, & L_{2}=-(\star \mathbf{d} g) \boldsymbol{\theta}^{3}-\boldsymbol{\omega} \boldsymbol{\theta}^{3}, \\
L_{1}=\left(f_{0}+g_{0}\right) \boldsymbol{\theta}^{2} \boldsymbol{\theta}^{3}, & L_{3}=(\star \mathbf{d} f) \boldsymbol{\theta}^{2}+\boldsymbol{\omega} \boldsymbol{\theta}^{2}
\end{array}
$$

and the Sparling 3-forms

$$
\begin{array}{ll}
S_{0}=\left(\left(f_{0}+g_{0}\right) \boldsymbol{\omega}+f_{1} \mathbf{d} g+f_{0}(\star \mathbf{d} g)\right) \boldsymbol{\theta}^{2} \boldsymbol{\theta}^{3}, & S_{2}=\boldsymbol{\omega} \mathbf{d} g \boldsymbol{\theta}^{3}, \\
S_{1}=\left(\left(f_{1}+g_{1}\right) \boldsymbol{\omega}+f_{0} \mathbf{d} g+f_{1}(\star \mathbf{d} g)\right) \boldsymbol{\theta}^{2} \boldsymbol{\theta}^{3}, & S_{3}=-\boldsymbol{\omega} \mathbf{d} f \boldsymbol{\theta}^{2} .
\end{array}
$$

Here, we have used the notation $\star \boldsymbol{\alpha}$ for the 1 -form $\alpha_{0} \boldsymbol{\theta}^{1}+\alpha_{1} \boldsymbol{\theta}^{0}$ given the 1 -form $\boldsymbol{\alpha}=\alpha_{0} \boldsymbol{\theta}^{0}+\alpha_{1} \boldsymbol{\theta}^{1}$. Inserting these expressions into the equations $\mathbf{d} L_{i}=S_{i}$ (and stripping off the common factor $\boldsymbol{\theta}^{2} \boldsymbol{\theta}^{3}, \boldsymbol{\theta}^{3}$ and $\boldsymbol{\theta}^{2}$, respectively) yields the four equations on $\mathcal{M}_{1}$

$$
\begin{gathered}
\mathbf{d}\left(f_{1}+g_{1}\right)+\left(f_{1}+g_{1}\right)(\mathbf{d} f+\mathbf{d} g)=\left(f_{0}+g_{0}\right) \boldsymbol{\omega}+f_{1} \mathbf{d} g+f_{0}(\star \mathbf{d} g), \\
\mathbf{d}\left(f_{0}+g_{0}\right)+\left(f_{0}+g_{0}\right)(\mathbf{d} f+\mathbf{d} g)=\left(f_{1}+g_{1}\right) \boldsymbol{\omega}+f_{0} \mathbf{d} g+f_{1}(\star \mathbf{d} g), \\
\mathbf{d}(\star \mathbf{d} g)+\mathbf{d} g(\star \mathbf{d} g)+\mathbf{d} \boldsymbol{\omega}=0, \\
\mathbf{d}(\star \mathbf{d} f)+\mathbf{d} f(\star \mathbf{d} f)+\mathbf{d} \boldsymbol{\omega}=0 .
\end{gathered}
$$

The first pair of equations are 1-form equations. They comprise four scalar equations. However, they contain $\boldsymbol{\omega}$ which indicates that they depend on the chosen 
gauge. We can extract two gauge invariant equations by multiplying with the basis 1 -forms and combining the equations linearly. This leads to the 2 -form equations

$$
\begin{array}{r}
\mathbf{d}^{2}(f+g)+\mathbf{d}(f+g) \mathbf{d}(f+g)=0, \\
\mathbf{d}(\star \mathbf{d} f)+\mathbf{d}(\star \mathbf{d} g)+\mathbf{d}(f+g)((\star \mathbf{d} f)+(\star \mathbf{d} g))=0 .
\end{array}
$$

The first of these is an identity while the second equation can be combined with the third and fourth equation of (41). So we have the following three 2-form equations

$$
\begin{gathered}
\mathbf{d} \boldsymbol{\omega}=\mathbf{d} f(\star \mathbf{d} g), \\
\mathbf{d}(\star \mathbf{d} g)+\mathbf{d} g(\star \mathbf{d} g)+\mathbf{d} f(\star \mathbf{d} g)=0, \\
\mathbf{d}(\star \mathbf{d} f)+\mathbf{d} f(\star \mathbf{d} f)+\mathbf{d} g(\star \mathbf{d} f)=0 .
\end{gathered}
$$

Note, that we do not attach a meaning to the forms $\star \mathbf{d} f$ and $\star \mathbf{d} g$ as yet. These symbols are so far only abbreviations for the forms $\star \mathbf{d} f=f_{0} \boldsymbol{\theta}^{1}+f_{1} \boldsymbol{\theta}^{0}$ and $\star \mathbf{d} g=$ $g_{0} \boldsymbol{\theta}^{1}+g_{1} \boldsymbol{\theta}^{0}$.

Thus, we end up with the following set of differential forms which we regard as being defined on the frame bundle over $\mathcal{M}_{1}$

$$
\begin{aligned}
\mathbf{Z}_{0} & \equiv \mathbf{d}\left(f_{0}+g_{0}\right)+\left(f_{0}+g_{0}\right)(\mathbf{d} f+\mathbf{d} g) \\
& -\left(f_{1}+g_{1}\right) \boldsymbol{\omega}-\left(f_{0} g_{0}+f_{1} g_{1}\right) \boldsymbol{\theta}^{0}-\left(f_{1} g_{0}+f_{0} g_{1}\right) \boldsymbol{\theta}^{1}, \\
\mathbf{Z}_{1} & \equiv \mathbf{d}\left(f_{1}+g_{1}\right)+\left(f_{1}+g_{1}\right)(\mathbf{d} f+\mathbf{d} g) \\
& -\left(f_{0}+g_{0}\right) \boldsymbol{\omega}-\left(f_{0} g_{0}+f_{1} g_{1}\right) \boldsymbol{\theta}^{1}-\left(f_{1} g_{0}+f_{0} g_{1}\right) \boldsymbol{\theta}^{0}, \\
\mathbf{Z}_{2} & \equiv \mathbf{d} \boldsymbol{\omega}-\mathbf{d} f(\star \mathbf{d} g) \\
\mathbf{Z}_{3} & \equiv \mathbf{d}(\star \mathbf{d} g)+\mathbf{d} g(\star \mathbf{d} g)+\mathbf{d} f(\star \mathbf{d} g), \\
\mathbf{Z}_{4} & \equiv \mathbf{d}(\star \mathbf{d} f)+\mathbf{d} f(\star \mathbf{d} f)+\mathbf{d} g(\star \mathbf{d} f), \\
\mathbf{Z}_{5} & \equiv \mathbf{d} \boldsymbol{\theta}^{0}+\boldsymbol{\omega} \boldsymbol{\theta}^{1}, \\
\mathbf{Z}_{6} & \equiv \mathbf{d} \boldsymbol{\theta}^{1}+\boldsymbol{\omega} \boldsymbol{\theta}^{0}, \\
\mathbf{Z}_{7} & \equiv \mathbf{d} f-f_{0} \boldsymbol{\theta}^{0}-f_{1} \boldsymbol{\theta}^{1}, \\
\mathbf{Z}_{8} & \equiv \mathbf{d} g-g_{0} \boldsymbol{\theta}^{0}-g_{1} \boldsymbol{\theta}^{1}, \\
\mathbf{Z}_{9} & \equiv\left(\mathbf{d} f_{0}-\boldsymbol{\omega} f_{1}\right) \boldsymbol{\theta}^{0}+\left(\mathbf{d} f_{1}-\boldsymbol{\omega} f_{0}\right) \boldsymbol{\theta}^{1}, \\
\mathbf{Z}_{10} & \equiv\left(\mathbf{d} g_{0}-\boldsymbol{\omega} g_{1}\right) \boldsymbol{\theta}^{0}+\left(\mathbf{d} g_{1}-\boldsymbol{\omega} g_{0}\right) \boldsymbol{\theta}^{1}, \\
\mathbf{Z}_{11} & \equiv \star \mathbf{d} f-f_{0} \boldsymbol{\theta}^{1}-f_{1} \boldsymbol{\theta}^{0}, \\
\mathbf{Z}_{12} & \equiv \star \mathbf{d} g-g_{0} \boldsymbol{\theta}^{1}-g_{1} \boldsymbol{\theta}^{0}, \\
\mathbf{Z}_{13} & \equiv \mathbf{d}(\star \mathbf{d} f)-\left(\mathbf{d} f_{0}-\boldsymbol{\omega} f_{1}\right) \boldsymbol{\theta}^{1}-\left(\mathbf{d} f_{1}-\boldsymbol{\omega} f_{0}\right) \boldsymbol{\theta}^{0}, \\
\mathbf{Z}_{14} & \equiv \mathbf{d}(\star \mathbf{d} g)-\left(\mathbf{d} g_{0}-\boldsymbol{\omega} g_{1}\right) \boldsymbol{\theta}^{1}-\left(\mathbf{d} g_{1}-\boldsymbol{\omega} g_{0}\right) \boldsymbol{\theta}^{0} .
\end{aligned}
$$

Apart from the forms arising from the Witten-Sparling equations this system includes forms which implement the expansion of $\mathbf{d} f, \mathbf{d} g, \star \mathbf{d} f$ and $\star \mathbf{d} g$ in terms of their components $f_{0}, f_{1}, g_{0}$ and $g_{1}$ as well as their exterior derivatives.

Note, that there is also the first Bianchi identity which should be added. It reads

$$
\mathrm{d} \boldsymbol{\omega} \boldsymbol{\theta}^{0}=0=\mathrm{d} \boldsymbol{\omega} \boldsymbol{\theta}^{1} .
$$

However, since it is an identity and, in addition, a 3-form equation which cannot be implemented in our 2-dimensional problem we will simply assume its general validity without listing it among the relevant forms. 
A closer and somewhat tedious examination of the system reveals the following facts:

$$
\begin{gathered}
\mathbf{d} \mathbf{Z}_{0} \in\left\langle\mathbf{Z}_{0}, \mathbf{Z}_{1}, \mathbf{Z}_{2}, \mathbf{Z}_{3}, \mathbf{Z}_{4}, \mathbf{Z}_{7}, \mathbf{Z}_{8}, \mathbf{Z}_{9}, \mathbf{Z}_{11}, \mathbf{Z}_{12}, \mathbf{Z}_{13}\right\rangle \\
\mathbf{d} \mathbf{Z}_{1} \in\left\langle\mathbf{Z}_{0}, \mathbf{Z}_{1}, \mathbf{Z}_{2}, \mathbf{Z}_{3}, \mathbf{Z}_{4}, \mathbf{Z}_{7}, \mathbf{Z}_{8}, \mathbf{Z}_{10}, \mathbf{Z}_{11}, \mathbf{Z}_{12}, \mathbf{Z}_{14}\right\rangle \\
\mathbf{d} \mathbf{Z}_{2} \in\left\langle\mathbf{Z}_{3}\right\rangle \cap\left\langle\mathbf{Z}_{4}\right\rangle, \quad \mathbf{d} \mathbf{Z}_{3} \in\left\langle\mathbf{Z}_{3}\right\rangle, \quad \mathbf{d} \mathbf{Z}_{4} \in\left\langle\mathbf{Z}_{4}\right\rangle, \\
\mathbf{d} \mathbf{Z}_{5} \in\left\langle\mathbf{Z}_{6}\right\rangle, \quad \mathbf{d Z}_{6} \in\left\langle\mathbf{Z}_{5}\right\rangle, \\
\mathbf{d Z}_{7} \in\left\langle\mathbf{Z}_{9}\right\rangle, \quad \mathbf{d} \mathbf{Z}_{8} \in\left\langle\mathbf{Z}_{10}\right\rangle, \quad \mathbf{d} \mathbf{Z}_{9} \in\left\langle\mathbf{Z}_{5}, \mathbf{Z}_{6}\right\rangle, \quad \mathbf{d} \mathbf{Z}_{10} \in\left\langle\mathbf{Z}_{5}, \mathbf{Z}_{6}\right\rangle, \\
\mathbf{d} \mathbf{Z}_{11} \in\left\langle\mathbf{Z}_{13}\right\rangle, \quad \mathbf{d} \mathbf{Z}_{12} \in\left\langle\mathbf{Z}_{14}\right\rangle, \quad \mathbf{d} \mathbf{Z}_{13} \in\left\langle\mathbf{Z}_{5}, \mathbf{Z}_{6}\right\rangle, \quad \mathbf{d} \mathbf{Z}_{14} \in\left\langle\mathbf{Z}_{5}, \mathbf{Z}_{6}\right\rangle .
\end{gathered}
$$

The crucial forms are $\mathbf{Z}_{2}, \ldots, \mathbf{Z}_{6} . \mathbf{Z}_{2}, \mathbf{Z}_{3}, \mathbf{Z}_{4}$ determine the connection and $\mathbf{Z}_{5}$ and $\mathbf{Z}_{6}$ determine the metric in terms of the frame $\boldsymbol{\theta}^{0}$ and $\boldsymbol{\theta}^{1}$. The forms $\mathbf{Z}_{7}, \ldots, \mathbf{Z}_{14}$ are 'book keeping' forms in the sense that they express $\mathbf{d} f, \mathbf{d} g, \star \mathbf{d} f$ and $\star \mathbf{d} g$ in terms of the components $f_{0}, f_{1}, g_{0}$ and $g_{1}$. These are not really necessary if we are only interested in the geometry of $\mathcal{M}_{1}$. In fact, in this case it is not even necessary to know about the functions $f$ and $g$.

We can extract several systems from these equations. The first system is obtained by ignoring that $f_{0}, \ldots, g_{1}$ are components of 1 -forms with respect to the coframe and working simply with these four functions. The system we use consists of $\mathbf{Z}_{0}, \mathbf{Z}_{1}, \mathbf{Z}_{5}, \mathbf{Z}_{6}$, as well as $\mathbf{Z}_{3}+\mathbf{Z}_{2}$ and $\mathbf{Z}_{4}+\mathbf{Z}_{2}$. In addition, we include $\mathbf{Z}_{9}$, i.e., the exterior derivative of $\mathbf{Z}_{7}$. The corresponding equation for $g_{0}$ and $g_{1}$ follows from $\mathbf{Z}_{0}$ and $\mathbf{Z}_{1}$. This is the system (77).

The second system is obtained by eliminating the components as completely as possible. We use generic 1-forms $\boldsymbol{\alpha}, \boldsymbol{\beta}, \boldsymbol{\gamma}$ and $\boldsymbol{\delta}$ for the 1 -forms $\mathbf{d} f, \star \mathbf{d} f, \mathbf{d} g$ and $\star \mathbf{d} g$, respectively. Then the system consists of $\mathbf{Z}_{0} \boldsymbol{\theta}^{0}+\mathbf{Z}_{1} \boldsymbol{\theta}^{1}$, linear combinations of $\mathbf{Z}_{2}, \mathbf{Z}_{3}$ and $\mathbf{Z}_{4}$ as well as $\mathbf{Z}_{5}$ and $\mathbf{Z}_{6}$. Finally we add the closedness of $\boldsymbol{\alpha}$ and $\boldsymbol{\gamma}$ and the algebraic relationships between $\boldsymbol{\alpha}$ and $\boldsymbol{\beta}$ respectively $\boldsymbol{\gamma}$ and $\boldsymbol{\delta}$. This results in the system (8).

Note, that it is enough to include the combination of $\mathbf{Z}_{0}$ and $\mathbf{Z}_{1}$ given above. This is due to the following consideration. Let $I=\left\langle\mathbf{Z}_{k}, 2 \leq k \leq 14\right\rangle$ be the ideal generated by all forms except for $\mathbf{Z}_{0}$ and $\mathbf{Z}_{1}$. Then we have

$$
\begin{array}{ll}
\mathbf{d} \mathbf{Z}_{0}-\boldsymbol{\omega} \mathbf{Z}_{1}+\mathbf{d}(f+g) \mathbf{Z}_{0} \equiv 0 & \bmod I, \\
\mathbf{d} \mathbf{Z}_{1}-\boldsymbol{\omega} \mathbf{Z}_{0}+\mathbf{d}(f+g) \mathbf{Z}_{1} \equiv 0 & \bmod I .
\end{array}
$$

By taking another exterior derivative we get the equations

$$
\begin{array}{ll}
\mathbf{d} \boldsymbol{\omega} \mathbf{Z}_{1} \equiv 0 & \bmod I, \\
\mathbf{d} \boldsymbol{\omega} \mathbf{Z}_{0} \equiv 0 & \bmod I,
\end{array}
$$

since $\mathbf{d} I \subset I$. This implies, that if all other equations hold, then we have $\mathbf{d} \boldsymbol{\omega} \mathbf{Z}_{i}=0$ which forces $\mathbf{Z}_{i}=0$ unless $\mathbf{d} \boldsymbol{\omega}=0$, a case in which we are not interested.

\section{REFERENCES}

[1] Alcubierre M. et al. 2004 Toward standard testbeds for numerical relativity. Class. Quant. Grav. 21 589. see also http://www.appleswithapples.org/

[2] Baumgarte T. W. and Shapiro S. L. 2003 Numerical Relativity and Compact Binaries. Physics Reports 37641.

[3] Berger B. K. 2001 Numerical Approaches to Spacetime Singularities. Living Rev. Relativity http://www.livingreviews.org/lrr-1998-7

[4] Bossavit A, 1998 Computational Electromagnetism (Boston: Academic Press). 
[5] Bossavit A, 1988 Mixed finite elements and the complex of Whitney forms. In The Mathematics of Finite Elements and Applications VI, ed. J Whiteman (London: Academic Press). pp. 137-144.

[6] Bossavit A, 1998-2000 Discretization of electromagnetic problems. Tech. rep., Interdyscyplinary Centre For Mathematical And Computational Modelling, Warsaw. Http://www.icm.edu.pl/edukacja/mat/DEP.php.

[7] Cartan É, 2001 Riemannian Geometry In An Orthogonal Frame (Singapore: World Scientific). Lectures delivered by E. Cartan at the Sorbonne 1926-27.

[8] Estabrook F. B. and Wahlquist H. D. 1989 Classical geometries defined by exterior differential systems on higher frame bundles. Class. Quant. Grav. 6 263-274.

[9] Frankel T, 1997 The geometry of Physics - An introduction (Cambridge: Cambridge University press)

[10] Frauendiener J, 2006 Discrete differential forms in General Relativity. Class. Quant. Grav. 23 S369-S385.

[11] Galassi M et al., 2005 GNU Scientific Library Reference Manual. http://www.gnu.org/software/gsl/manual/gsl-ref_toc.html

[12] Garfinkle D 2002 Harmonic coordinate method for simulating generic singularities. Phys. Rev. D 65044029.

[13] Gentle A P, 2002 Regge calculus: a unique tool for numerical relativity. Gen. Rel. Grav. 34 $1701-1718$.

[14] Gomez R, Lehner L, Papadopoulos P and Winicour J 1997 The eth formalism in numerical relativity. Class. Quant. Grav. 14 977-990

[15] Gowdy R H 1971 Gravitational Waves in Closed Universes. Phys. Rev. Lett. 27826. Gowdy R H 1974 Vacuum Space-Times with two Parameter Spacelike Isometry Groups and Compact Invariant Hypersurfaces: Topologies and Boundary Conditions. Ann. Phys. 83203.

[16] Hiptmair R 2002 Finite elements in computational electromagnetism. Acta Numerica 11 237-339.

[17] Kuranishi M 1957 On E. Cartan's Prolongation Theorem of Exterior Differential Systems. Amer. J. Math. 79 1-47.

[18] Landau, L D and Lifschitz, E F, 1975 The Classical Theory of Fields (Oxford: Pergamon Press)

[19] Munkres J R, 1993 Simplicial complexes and simplicial maps. In Elements of Algebraic Topology (Perseus Press), chap. 1.2. pp. 7-14.

[20] Nédélec J C, 1980 Mixed finite elements in $\mathbb{R}^{3}$. Numer. Math. 35 315-341.

[21] New K C B, Watt K, Misner C W and Centrella J M 1998 Stable 3-level leapfrog integration in numerical relativity. Phys. Rev. D 58064022.

[22] Oteo J. A. 1991 The Baker-Campbell-Hausdorff Formula and Nested Commutator Identities. J. Math. Phys. 32589.

[23] Winicour J et al. 2004 Apples with Apples results. Tech. rep., Department of Physics and Astronomy, University of Pittsburgh http://artemis.phyast.pitt.edu/.

[24] Powell M J D 1970 A hybrid method for nonlinear equations. In Numerical Methods for Nonlinear Algebraic Equations, ed. P Rabinowitz (Gordon and Breach).

[25] van Putten M H 1997 Numerical integration of nonlinear wave equations for general relativity. Phys. Rev. D 554705.

[26] Raviart P A and Thomas J M, 1977 A mixed finite element method for 2nd order elliptic problems. In Mathematical aspects of the finite element method, eds. I Galligani and E Magenes (Berlin and New York: Springer-Verlag), vol. 606 of Lecture Notes in Mathematics.

[27] Regge T, 1961 General Relativity without coordinates. Nouvo Cimento 19 558-571.

[28] Richter R, Frauendiener J and Vogel M 2007 Application of Discrete Differential Forms to Spherically Symmetric Systems in General Relativity. Class. Quant. Grav. 24433.

[29] Richter R, Geometric Discretisations of General Relativity, PhD thesis, University of Tübingen, to be published.

[30] Scheel M A et al. 2006 Solving Einstein's Equations With Dual Coordinate Frames. Phys. Rev. D $\mathbf{7 4} 104006$.

[31] Schnetter E et al. 2006 A multi-block infrastructure for three-dimensional time-dependent numerical relativity. Class. Quant. Grav. 23 S553 - S578 
[32] Sopuerta C F and Laguna P 2006 Finite element computation of the gravitational radiation emitted by a pointlike object orbiting a nonrotating black hole. Phys. Rev. D 73044028.

[33] Sparling G, 2001 Twistors, spinors and the Einstein equations. In Further advances in twistor theory III: Curved twistor spaces, eds. L J Mason, L P Hughston, P Z Kobak and K Pulverer (Boca Raton: Chapman and Hall). pp 179-187.

[34] Spivak M 1979 A comprehensive introduction to differential geometry volume V (Publish or Perish), pp 158-179.

[35] Thornburg J 2004 Black-hole excision with multiple grid patches. Class. Quant. Grav. 21 3665-3691

[36] Whitney H 1957 Geometric Integration Theory (Princeton Univ. Press, Princeton).

E-mail address: richter@na.uni-tuebingen.de

Mathematisches Institut, Universität Tübingen, Auf der Morgenstelle 10, 72076 Tübingen, Germany

E-mail address: joergf@maths.otago.ac.nz

Centre of Mathematics for Applications, University of Oslo, P.O. Box 1053, BlinDERN, NO-0316 OSLO, NoRWAY

Current address: Department of Mathematics and Statistics, University of Otago, P.O. Box 56, Dunedin 9010, New Zealand 